\title{
WILLINGNESS TO PAY FOR CONNECTED VEHICLES: AN ALTERNATIVE-SPECIFIC MIXED LOGIT REGRESSION APPROACH
}

\author{
Amirreza Nickkar1, Hyeon-Shic Shin'², Young-Jae Lee ${ }^{3}$ \\ 1,3 Department of Transportation and Urban Infrastructure Studies, Morgan State University, Baltimore, USA \\ ${ }^{2}$ Department of City and Regional Planning, Morgan State University, Baltimore, USA
}

Received 3 February 2020; accepted 20 March 2020

\begin{abstract}
The study of connected vehicles (CVs) has become a hot topic in recent years. Understanding the characteristics that lead consumers to relate to CVs motivates researchers to conduct market analysis studies. The current research investigated the socio-demographic attributes that may contribute to the individual preferences for purchasing CVs. Researchers constructed a series of Alternative-Specific Mixed Logit models to examine the associations between individual preferences of respondents and their willingness to pay for CV features in their future vehicle. The results indicate that hours spent driving play a privileged role among sociodemographic characteristics and driving behavior attributes of respondents. People who drive longer hours tended to purchase CV features. Also, the factor of age had a noticeable effect as the results showed that older people are more likely to purchase CV features.
\end{abstract}

Keywords: automated mobility, connected autonomous vehicle, willingness to pay, adoption, mixed Logit model.

\section{Introduction}

Both scholars and transit operators are concentrating on the development of connected vehicles (CVs), along with rapid technological developments and emerging innovative mobility methods. CVs' most expected advantages are improving traffic safety levels and the performance of the transportation network (Arvin et al., 2019; Dowling et al., 2016; Ghiasi et al., 2019; Kidando et al., 2018; Li et al., 2016). The Internet of Things (IoT), as one of the most remarkable technological developments in the recent decade, provides connectivity for all mobile applicants and fixed infrastructures at any time and any location. The automotive industry can take advantage of this revolutionary technological innovation to design the next generation of vehicles. The connectivity of transportation elements, through sharing the real-time information, will dramatically improve the performance, efficiency and safety of whole transportation systems (Andorka and Rambow-Hoeschele, 2020). The connectivity and ability to transmit data in the real environment can be established between a vehicle and other vehicles (V2V) and vehicle to infrastructures (V2I) through the IoT. The IoT introduces a new feature of "V2X" that enables communications between the $\mathrm{CV}$ and other features that potentially can change the vehicle guidance (i.e., vehicles, infrastructures, pedestrians, etc.) (Guerrero-ibanez et al., 2015).

${ }^{1}$ Corresponding author: amirreza.nickkar@morgan.edu 
A remarkable trend of investing in the connected vehicles' market has occurred in recent years. Estimations show that revenue from the monetization of car data will be over $\$ 400$ billion by 2030 (Van Themsche, 2016). In the United States, the U.S. Department of Transportation (USDOT) and all state DOTs have shown extreme interest in developing $\mathrm{CV}$ technology and its infrastructures (Bertini et al., 2016). The USDOT in collaboration with the Society of Automotive Engineers (SAE) has already began determining V2V and V2I communication regulations and standards while previous estimations showed over 100 million vehicles in the US will be embedded with telematics by 2025 (Bock et al., 2016). The National Highway Traffic Safety Administration (NHTSA) categorized connected automated vehicles (CAVs) into five levels from level 0 to level 4 in which level 0 represents vehicles that are controlled by drivers and level 4 reflects vehicles that are fully automated and the vehicle itself can manage all driving tasks. The NHTSA, recently, stated that the V2V and V2I connectivity features can potentially eliminate $80 \%$ of all unimpaired crashes' scenarios (Narla, 2013). This emphasis on CV technology has motivated many researchers to conduct analytical studies to measure the readiness of the automotive market to embrace these emerging vehicles. Several studies in recent years have been conducted to understand the demographic characteristics of people that relate to their preferences for purchasing and using advanced features in their future vehicles. Since information transmission and safety assistance are the main pillars of $\mathrm{CV}$ technology, it would very helpful to better understand the possible relationship between sociodemographic characteristics of potential users of CVs and their willingness-to-use (WTU) preferences for $\mathrm{CV}$ features. Moreover, this knowledge will help us understand penetration levels of these features in the future markets (Kopelias et al., 2019).

This study aims to identify individuals' preferences for $\mathrm{CV}$ features using an appropriate statistical approach. Therefore, this study attempts to answer this question: What are the demographic characteristics and travel behavior attributes related to people's preferences and WTP for CVs? To undertake this analysis, an online survey has been conducted in the United States from September 2013 to April 2014 to collect socioeconomic attributes and choice behaviors of drivers for purchasing a CV. The contribution of this study is to find possible relationships between sociodemographic characteristics of participants and their WTP when purchasing connected vehicles in the future by using an appropriate and sophisticated statistical analysis method. The organization of the paper is as follows. Section 2 provides an overview of past related studies and proposes shortcomings in the background research that will be addressed in the current study. Section 3 presents an analysis methodology of the study and overall results of the conducted survey. Section 4 provides results of the statistical analysis, and, finally, discussion and conclusions are presented in Section 5. The results of this study could be utilized by auto market analysts, transportation economists, transportation authorities, transport investment agencies, and collaborators in emerging and advanced transportation systems.

\section{Literature Review}

As discussed in the introduction section, CV focus on increasing safety levels and assisting drivers by providing real-time information. In recent years many studies covered the issues of 
willingness-to-pay (WTP) and willingness-touse (WTU) for vehicles with automated and advanced features. This section tries to cover past studies related to sociodemographic characteristics' factors that may influence WTP and WTU for such vehicles.

According to the past studies, various attributes of people may influence WTP and WTU for advanced features in a vehicle. Level of risk taking, social trust, convenience and satisfaction issues, price, environmental concerns, incentives or discounts, luxury, and efficiency are the most important attributes revealed in the past studies as motivating factors for purchasing and using a new advanced feature in the vehicle (Choi and Koo, 2019; Jiang et al., 2018; Kyriakidis et al., 2015; Liu et al., 2019a, Motamedi et al., 2018; Sahebi and Nassiri, 2017; Schoettle and Sivak, 2014; Shabanpour et al., 2018). A study by Bansal and Kockelman (2017) showed that almost half of the people in the United States are willing to pay extra money for automated features in their next vehicle and obviously people with higher income levels have a greater tendency to do so. Among other characteristics of people willing to use advanced features in their next vehicle, age and gender showed significant relationships. People who are early adopters (Zmud et al., 2016b), male (Bansal et al., 2016; Jiang et al., 2018) and younger (Abraham et al., 2017; Payre et al., 2014; Wang et al., 2019) have shown more WTU for advanced and automated vehicles. Also, some studies proved that eagerness to purchase and use automation technologies has a direct relationship with the education attainment and the level of knowledge about emerging vehicles (Daziano et al., 2017; Ebnali et al., 2019; Shin et al., 2015; Zmud et al., 2016a). Purchasing and using automated and emerging features in the vehicle may also impact travel behavior and commuting patterns of the drivers, causing them to increase vehicle mile traveled (VMT) or, inversely, forcing drivers to take shorter distances (Gkartzonikas and Gkritza, 2019; Shin et al., 2019).

Among those mentioned motivating attributes for purchasing and using advanced features in the vehicle, the price of the feature may play a more critical role (Shabanpour et al., 2017). Daziano et al. (2017) explored that people generally are willing to use automated electric vehicles if the vehicle is able to drive a longer range; however, they are concerned about the price of the vehicle and fuel costs. The safety level of the CV's features is the other important attribute that influences people's decision in purchasing and using them. Basically, two main factors of optimal design and reliability determine the effectiveness of safety features in CVs. The design of safety features meets the driver's need for an automated feature that warns of and reacts to a hazard in a timely manner, and the reliability of the feature increases the driver's comfort because they are confident, they have control over unpredicted conditions (Medenica, 2019). However, the various connectivity features that will be introduced in the automotive market will have issues such as privacy and security sensitivity, data transmission approaches, and ethical frameworks, which also will be subject to competition (Outay et al., 2017).

Regarding implemented methodologies, stated preference methods were the main approaches for measuring WTP for CVs. Discrete choice modeling using Logit models and a fixed-response forced-choice method were the main approaches that have been used widely in past studies to evaluate preferences of future buyers of CVs (Abraham et al., 2017; Bansal et al., 2016; Daziano et al., 2017). Also, 
some studies used the contingent valuation method to determine the amount people are willing to pay for $\mathrm{CV}$ attributes (Kyriakidis et al., 2015; Liu et al., 2019a, Liu et al., 2019b, Schoettle and Sivak, 2014). Obviously, Logit models were used the most in past stated preference studies on this topic; however, new approaches of the Logit model should be implemented to identify homogenous and heterogeneous variables in the dataset which is not possible with standard types of the Logit model. Therefore, this study uses an Alternative-Specific Mixed Logit Regression approach to incorporate heterogeneity considerations.

\section{Methodology}

The current study undertakes the stated preferences choice modeling methodology. An Alternative-Specific Mixed Logit Regression Model or Mixed Logit Model (MXL) has been utilized due to its more flexible heterogeneous capability to provide more realistic choice patterns regarding the factors that influence respondents' final decisions (Jaffry and Apostolakis, 2011).

\subsection{Survey}

The data collection process has been performed based on an online survey. The survey had two main components: sociodemographic data and attributes of purchasing an autonomous vehicle. The researchers designed the survey using Sawtooth software (Sawtooth Software, 2009) which is, by consensus, the leading provider of conjoint analysis software. The survey links were promoted nationwide in the U.S. through advertising websites (such as craigslist) and social media networks. The survey was administered from September 2013 through April 2014; and 529 usable responses were received.
The data has been archived and preserved electronically. All participants first received explanations about the goal of the survey and confidential issues in the survey's first screen. The survey was divided into three sections: first, the most important socioeconomic characteristics (e.g., gender, age, and the number of adults and children under 18 in the household), last vehicle purchase/lease experience, and current spatial pattern of commuting trips. The survey also asked participants about the minimum and maximum amount of money that they will pay for a new vehicle. Drivers were then asked the level of knowledge about the autonomous vehicles. The next section focused on drivers' stated preferences for purchasing CVs. Participants were first provided with a description of the different levels of CVs according to the definitions of the National Highway Traffic Safety Administration (NHTSA) for CVs (National Highway Traffic Safety Administration, 2018).

\subsection{Survey}

After the data collection process, 529 valid responses were implemented for analysis. A summary of selected socioeconomic variables is presented in Table 1 . Gender was balanced, with $51.2 \%$ male and $48.8 \%$ female respondents, which is not much different from the national average ( 0.97 male/female) (United States Census Bureau, 2017). The age distribution also reflected the national statistics.

As to race/ethnicity, Whites were somewhat similar to the national average, about $65.6 \%$. With respect to education, $29.5 \%$ had graduate-level academic degrees. This is a little higher than the national average of $28.1 \%$ (United States Census Bureau, 2017). The overrepresentation of these 
highly educated participants might be due in part to the recruitment method, as many participants were from the Morgan State University and research centers in various academic institutions. While the median household income was $\$ 61,372$ in 2017 (United States Census Bureau, 2017), the number of respondents in each income group was balanced. Other socioeconomic variables were also compared to the national statistics. The distributions of demographic characteristics were generally similar to the national statistics, making our sample relatively representative.

\section{Table 1}

Summary of Participants' Characteristics

\begin{tabular}{|c|c|c|c|}
\hline Demographics & Characteristics & Study $(\%)^{3}$ & U.S. $(\%)^{3}$ \\
\hline \multirow{2}{*}{ Gender $^{1}$} & Male & 51.2 & 49.2 \\
\hline & Female & 48.8 & 50.8 \\
\hline \multirow{5}{*}{ Age $^{1}$} & Less than 30 & 21.4 & 21.8 \\
\hline & $30-39$ & 21.6 & 16.9 \\
\hline & $40-49$ & 22.9 & 17.7 \\
\hline & $50-59$ & 21.4 & 17.9 \\
\hline & 60 and more & 12.9 & 16 \\
\hline \multirow{5}{*}{ Race/ethnicity ${ }^{1}$} & White (Non-Hispanic) & 65.6 & 67.9 \\
\hline & Hispanic & 5.1 & 14.4 \\
\hline & Black/African-American & 17.3 & 11.8 \\
\hline & Asian & 5.9 & 4.9 \\
\hline & Other & 32 & 1 \\
\hline \multirow{4}{*}{ Education $^{1}$} & Associate degree and lower & 38.5 & 25.7 \\
\hline & Bachelor's degree & 31.9 & 20.5 \\
\hline & Master's degree & 19.5 & 23.0 \\
\hline & Doctoral or postdoctoral degree & 10.1 & 30.9 \\
\hline \multirow{3}{*}{ Household annual income ${ }^{1}$} & Less than $\$ 50,000$ & 36.1 & 46.5 \\
\hline & $\$ 50,000-\$ 99,999$ & 32.4 & 29.9 \\
\hline & $\$ 100,000$ and more & 31.5 & 23.6 \\
\hline \multirow{3}{*}{ Household size $^{1}$} & 1 & 5.3 & 27.6 \\
\hline & 2 & 30.2 & 33.7 \\
\hline & 3 and more & 64.5 & 38.7 \\
\hline \multirow{5}{*}{ Type of current vehicle ${ }^{2}$} & Sedan or coupe & 44.4 & 49.0 \\
\hline & SUV & 21.1 & 10.6 \\
\hline & Van & 5.4 & 3.5 \\
\hline & Truck SUV & 7.1 & 23.6 \\
\hline & Other & 28.6 & 13.3 \\
\hline \multirow{5}{*}{$\begin{array}{l}\text { Total driving time } \\
\text { during a day }\end{array}$} & Less than 0.5 hour & 0.11 & - \\
\hline & 0.5 to 1 hour & 0.29 & - \\
\hline & 1 to 1.5 hours & 0.28 & - \\
\hline & 1.5 to 2 hours & 0.22 & - \\
\hline & More than 2 hours & 0.10 & - \\
\hline
\end{tabular}

${ }^{1}$ 2011-2015 American Community Survey 5 Year Estimates for the U.S. values

${ }^{2}$ National Transportation Statistics 2018; The U.S. Department of Transportation's Bureau of Transportation Statistics

${ }^{3}$ Percentages may not total 100 due to rounding 


\subsection{Explanation of Attributes}

After comprehensive studies and examinations of past studies on the issues of willingness to pay for emerging and advanced transportation, especially successful incentives for promoting using battery electric vehicles (as advanced vehicles) in the U.S, five main attributes have been selected - collision warning, driver assistance, enhanced safety, roadway information, and travel assistance - as the main attributes that may influence the decision of drivers in purchasing a connected autonomous vehicle. The questions and attributes were verified by researching current purchasing incentives and rebates of the main automobile manufacturers in the U.S., interviewing related experts, and investigating related published studies (Shin et al., 2016). Although researchers designed attributes of the survey based on the results of expert consultations and pretests, participants were able to comment about their experiment and provide feedback. The design of questions is based on answering this question: What type of $\mathrm{CV}$ do respondents want to buy as their next vehicle when the $\mathrm{CV}$ is an available personal vehicle in the market? Of course, the $\mathrm{CV}$ is not currently available in the market and incentive policies for selling CVs might differ from current incentive policies for internal combustion engine vehicles (ICEVs); however, perceptible attributes and levels in the survey would help respondents engage more with the survey and also provide policy makers and market economists with more understandable outcomes. This study also found that the minimum and maximum value to spend on the next vehicle is $\$ 16,377$ to $\$ 27,605$ (Shin et al., 2015).

\subsubsection{Collision Package}

The survey asked respondents what type of collision warning and prevention features they want to have in their next vehicle. The survey considered four levels for this attribute according to the current engine type of vehicles on the market: front collision warning, side collision warning, front and side collision warning, all-around collision warning. There is interest in the automotive market for purchasing collision warning systems, but some factors including price are still the main barriers to using these advance features (Razaob et al., 2019). This study adds $\$ 0$ for selecting "nothing," \$350 for selecting "front collision warning" (level 2), \$600 for selecting "side collision warning" (level 3), $\$ 900$ for selecting "front and side collision warning" (level 4) and \$1,100 for selecting “all-around collision warning" (level 5).

\subsubsection{Driver Assistance}

Surveying this attribute illustrated to what degree respondents were willing to pay for automation and driving assistance. As Bansal and Kockelman (2017) explored, there is no intention in the U.S. to pay big extra money for any of the advanced automation technology; therefore, the extra money for automation of the vehicle should be at a reasonable price that makes the survey realistic for the respondent. According to the reviewed studies and the current automotive market, the study considered $\$ 0$ for selecting "nothing," $\$ 600$ for an extra for "lane departure system" (level 2), \$750 for "intersection and left turn assist system" (level 3) and \$1,000 for "pedestrian and cyclist alert and do not pass warning" (level 4). 


\subsubsection{Enhanced Safety Package}

This attribute focused on pedestrian and cyclist detection and a collision avoidance feature that is an important safety factor when purchasing the next CV. This study, after reviewing designed automated safety features of main car manufacturers in the U.S., categorized enhanced safety packages into three categories. Therefore, the participants pay an extra $\$ 300$ for selecting “do not pass warning" (level 2), \$750 for selecting "pedestrian and cyclist alert" (level 3), \$750 for selecting "pedestrian and cyclist alert and do not pass warning system" (level 4).

\subsubsection{Roadway Information Package}

Basically, roadway information features will improve drivers' travel behavior by providing real-time data. Questions about this attribute aimed to assess how much providing roadway information automatically may affect preferences of customers in purchasing the CV. Sayer et al. (2007) stated that providing roadway information systems may affect the drivers' travel pattern. Therefore, this attribute included four levels: $\$ 0$ for selecting “nothing," \$300 for selecting "slow/stop/ wrong-way vehicle advisor” (level 2), \$300 for selecting "road condition notification" (level 3), and $\$ 500$ for selecting "road condition notification and slow/stop/wrongway vehicle advisor" (level 4).

\subsubsection{Travel Assistance Package}

Questions about this attribute concentrated on determining which group of participants is more interested in using travel assist systems. The travel assistance features help drivers to drive more smoothly. After reviewing current promotions in the automotive market, four different levels have been designed for this attribute: \$0 for selecting “nothing," \$250 for selecting "real time travel planning \& route optimization” (level 2), \$500 for selecting "parking spot locator" (level 3), and $\$ 700$ for selecting "real time travel planning \& route optimization and parking spot locator" (level 4).

\subsection{Alternative-Specific Mixed Logit Model}

In this section the structure of the utilized MXL model will be discussed. The index $d(d=1,2, \ldots, \mathrm{D})$ has been considered for participants, $f$ for the features $(f=1,2, \ldots$, F) and $\mathrm{c}$ for the choice occasion. Therefore, the utility of the individual $d$ associates with the features $f$ on choice occasion $c$ can be written as equation (1):

$U_{d f c}=\left(\gamma+\lambda_{d}\right) Z_{d f c}+\zeta_{d f c}$

Where $Z_{d f c}$ is a vector of feature attributes and the interactions of attributes among themselves and with respondent's characteristics, affecting the utility of individual $d$ for feature $f$ at the $c$ th choice occasion. $\gamma$ is a corresponding vector of the mean effects of the coefficients of $Z_{d f c}$ on feature choice and $\lambda_{d}$ is a vector with its $m$ th element representing unobserved factors specific to individual d. $\zeta_{d f c}$ represents a choice-occasion specific idiosyncratic random error term assumed to be identically and independently standard Gumbel distributed (Sener et al., 2009).

Therefore, in this study, the features that have been selected by participants were 
considered as the dependent variable. The feature value in each attribute was considered as the independent variable and the features were considered as the alternative variable. Obviously, the sociodemographic characteristics and travel behavior attributes of respondents were selected as the case-specific variables. As a result, researchers conducted five separate MXL models.

\section{Results}

The probabilities were estimated as a function of participants' socioeconomic characteristics. All statistical analyses were conducted using STATA 15. Statistical significance was evaluated at $0.1,0.05$, and 0.01 probability levels. The feature of "nothing" (level 1) was selected as the reference category in all models.

The first MXL model is related to features of the collision attribute. Two variables of driving hours and education attainment were statistically significant in the model. As shown in Table 2, higher educational attainment and more driving hours in a day are related to choosing "front and side collision warning systems”.

Table 2

Summarized the MXL Model Results for Attribute 1

\begin{tabular}{|c|c|c|c|c|c|c|c|}
\hline Attribute $^{1}$ & Feature & Coef. $^{2}$ & Std.Err ${ }^{3}$ & $\mathbf{z}$ & $\mathbf{P}>|\mathbf{z}|$ & \multicolumn{2}{|c|}{ [95\% Conf. Interval] } \\
\hline \multirow{3}{*}{ Level 2} & Driving hours & 0.24 & 0.14 & 1.68 & 0.092 & -0.04 & 0.53 \\
\hline & Education & 0.22 & 0.13 & 1.7 & 0.09 & -0.03 & 0.47 \\
\hline & Constant & -2.09 & 0.72 & -2.89 & 0.004 & -3.51 & -0.67 \\
\hline \multirow{3}{*}{ Level 3} & Driving hours & 0.45 & 0.23 & 1.96 & 0.05 & 0.00 & 0.89 \\
\hline & Education & 0.37 & 0.21 & 1.76 & 0.078 & -0.04 & 0.79 \\
\hline & Constant & -4.61 & 1.26 & -3.67 & 0 & -7.07 & -2.14 \\
\hline \multirow{3}{*}{ Level 4} & Driving hours & -0.02 & 0.17 & -0.11 & 0.914 & -0.35 & 0.31 \\
\hline & Education & 0.42 & 0.15 & 2.72 & 0.006 & 0.12 & 0.72 \\
\hline & Constant & -2.61 & 0.86 & -3.02 & 0.003 & -4.30 & -0.92 \\
\hline \multirow{3}{*}{ Level 5} & Driving hours & 0.33 & 0.12 & 2.8 & 0.005 & 0.10 & 0.55 \\
\hline & Education & 0.13 & 0.10 & 1.32 & 0.187 & -0.06 & 0.33 \\
\hline & Constant & -1.10 & 0.56 & -1.96 & 0.05 & -2.21 & 0.00 \\
\hline
\end{tabular}

${ }^{1} \log$ likelihood $=-490.67$, Prob $>$ Chi2 $=0.0079,{ }^{2}$ Coefficient, ${ }^{3}$ Standard Error

The second MXL model is related to features of the driver assistance attribute. Three variables of age, driving hours and education attainment were statistically significant in the model. As shown in Table 3, older people and drivers with more driving hours in a day are related to choosing "Lane Departure System and Intersection \& Left Turn Assist”. 
Table 3

Summarized the MXL Model Results for Attribute 2

\begin{tabular}{|c|c|c|c|c|c|c|c|}
\hline Attribute $^{1}$ & Feature & Coef. & Std.Err & $\mathbf{z}$ & \multicolumn{2}{|c|}{$\mathbf{P}>|\mathbf{z}|$} & \multicolumn{2}{|c|}{$[\mathbf{9 5 \%}$ Conf. Interval $]$} \\
\hline \multirow{3}{*}{ Level 2 } & Age & 0.18 & 0.09 & 1.97 & 0.048 & 0.00 & 0.36 \\
\cline { 2 - 8 } & Driving hours & 0.22 & 0.12 & 1.9 & 0.057 & -0.01 & 0.45 \\
\cline { 2 - 8 } & Constant & -1.54 & 0.85 & -1.82 & 0.069 & -3.20 & 0.12 \\
\hline \multirow{3}{*}{ Level 3 } & Age & 0.31 & 0.16 & 1.96 & 0.05 & 0.00 & 0.62 \\
\cline { 2 - 8 } & Driving hours & 0.45 & 0.19 & 2.32 & 0.021 & 0.07 & 0.83 \\
\cline { 2 - 8 } & Constant & -3.85 & 1.47 & -2.62 & 0.009 & -6.72 & -0.97 \\
\hline \multirow{3}{*}{ Level 4 } & Age & 0.22 & 0.10 & 2.28 & 0.023 & 0.03 & 0.41 \\
\cline { 2 - 8 } & Driving hours & 0.26 & 0.12 & 2.07 & 0.038 & 0.01 & 0.50 \\
\cline { 2 - 8 } & Constant & -1.60 & 0.90 & -1.78 & 0.076 & -3.37 & 0.17 \\
\hline
\end{tabular}

${ }^{1} \log$ likelihood $=-413.61$, Prob $>$ Chi2 $=0.0091$

The third MXL model is related to features of the enhanced safety attribute. Two variables of age and education attainment were statistically significant in the model. As shown in Table 4, older people and drivers with more driving hours in a day are related to choosing "Pedestrian \& Cyclist Alert and Do Not Pass Warning”.

Table 4

Summarized the MXL Model Results for Attribute 3

\begin{tabular}{|c|c|c|c|c|c|c|c|}
\hline Attribute $^{1}$ & Feature & Coef. & Std.Err & $\mathbf{z}$ & \multicolumn{2}{|c|}{$\mathbf{P}>|\mathbf{z}|$} & \multicolumn{2}{|c|}{$[\mathbf{9 5 \%}$ Conf. Interval $]$} \\
\hline \multirow{4}{*}{ Level 2 } & Age & -0.01 & 0.10 & -0.11 & 0.914 & -0.22 & 0.19 \\
\cline { 2 - 9 } & Education & -0.05 & 0.12 & -0.41 & 0.681 & -0.29 & 0.19 \\
\cline { 2 - 9 } & Constant & -0.93 & 0.64 & -1.46 & 0.143 & -2.18 & 0.32 \\
\hline \multirow{4}{*}{ Level 3 } & Age & 0.21 & 0.10 & 2.1 & 0.036 & 0.01 & 0.41 \\
\cline { 2 - 9 } & Education & 0.22 & 0.12 & 1.81 & 0.07 & -0.02 & 0.45 \\
\cline { 2 - 9 } & Constant & -2.92 & 0.68 & -4.27 & 0 & -4.26 & -1.58 \\
\hline \multirow{3}{*}{ Level 4 } & Age & 0.09 & 0.09 & 1.03 & 0.305 & -0.08 & 0.26 \\
\cline { 2 - 9 } & Education & -0.13 & 0.10 & -1.3 & 0.192 & -0.33 & 0.07 \\
\cline { 2 - 9 } & Constant & -0.60 & 0.54 & -1.11 & 0.269 & -1.66 & 0.46 \\
\hline
\end{tabular}

${ }^{1} \log$ likelihood $=-461.51$, Prob $>$ Chi2 $=0.0372$

The fourth MXL model is related to features of the roadway information attribute. Two variables of household size ("Household Size) and maximum value for next vehicle (Max Paying) were statistically significant in the model. As shown in Table 5, people with bigger household size and drivers with higher maximum value for purchasing their next vehicle are related to choosing "Road Condition Notification and Slow/Stop/ Wrong-Way Vehicle Advisor". 
Table 5

Summarized the MXL Model Results for Attribute 4

\begin{tabular}{|c|c|c|c|c|c|c|c|}
\hline Attribute $^{1}$ & Feature & Coef. & Std.Err & $\mathbf{z}$ & \multicolumn{2}{|c|}{$\mathbf{P}>|\mathbf{z}|$} & \multicolumn{2}{|c|}{$[\mathbf{9 5} \%$ Conf. Interval $]$} \\
\hline \multirow{4}{*}{ Level 2 } & Household Size & -0.02 & 0.10 & -0.17 & 0.865 & -0.20 & 0.17 \\
\cline { 2 - 8 } & Driving hours & 0.16 & 0.12 & 1.42 & 0.154 & -0.06 & 0.39 \\
\cline { 2 - 8 } & Max Paying & 0.08 & 0.05 & 1.74 & 0.082 & -0.01 & 0.18 \\
\cline { 2 - 9 } & Constant & -1.56 & 0.50 & -3.11 & 0.002 & -2.55 & -0.58 \\
\hline \multirow{5}{*}{ Level 3 } & Household Size & -0.43 & 0.24 & -1.82 & 0.07 & -0.89 & 0.03 \\
\cline { 2 - 9 } & Driving hours & 0.01 & 0.22 & 0.06 & 0.954 & -0.42 & 0.45 \\
\cline { 2 - 9 } & Max Paying & 0.19 & 0.07 & 2.72 & 0.006 & 0.05 & 0.33 \\
\cline { 2 - 8 } & Constant & -2.36 & 0.90 & -2.62 & 0.009 & -4.12 & -0.60 \\
\hline \multirow{5}{*}{ Level 4 } & Household Size & 0.07 & 0.09 & 0.82 & 0.411 & -0.10 & 0.25 \\
\cline { 2 - 8 } & Driving hours & 0.22 & 0.11 & 1.94 & 0.053 & 0.00 & 0.44 \\
\cline { 2 - 8 } & Max Paying & 0.12 & 0.04 & 2.62 & 0.009 & 0.03 & 0.21 \\
\cline { 2 - 8 } & Constant & -2.10 & 0.50 & -4.21 & 0 & -3.08 & -1.12 \\
\hline
\end{tabular}

${ }^{1} \log$ likelihood $=-416.72$, Prob $>$ Chi2 $=0.0234$

The last MXL model is related to features of the travel assistance attribute. Two variables of age and driving hours were statistically significant in the model. As shown in Table 6 , younger people are more likely to select the "real time travel planning \& route optimization and parking spot locator" feature than older ones; however, those who drive shorter hours are more likely to select the feature of "parking spot locator".

Table 6

Summarized the MXL Model Results for Attribute 5

\begin{tabular}{|c|c|c|c|c|c|c|c|}
\hline Attribute $^{1}$ & Feature & Coef. & Std.Err & $\mathbf{z}$ & \multicolumn{2}{|c|}{$\mathbf{P}>|\mathbf{z}|$} & \multicolumn{2}{|c|}{$[\mathbf{9 5 \%}$ Conf. Interval] } \\
\hline \multirow{4}{*}{ Level 2 } & Age & -0.10 & 0.09 & -1.15 & 0.251 & -0.28 & 0.07 \\
\cline { 2 - 9 } & Driving hours & 0.06 & 0.14 & 0.42 & 0.677 & -0.22 & 0.34 \\
\cline { 2 - 9 } & Constant & -0.78 & 0.59 & -1.33 & 0.184 & -1.93 & 0.37 \\
\hline \multirow{4}{*}{ Level 3 } & Age & -0.51 & 0.15 & -3.35 & 0.001 & -0.81 & -0.21 \\
\cline { 2 - 9 } & Driving hours & 0.42 & 0.21 & 1.97 & 0.049 & 0.00 & 0.84 \\
\cline { 2 - 9 } & Constant & 0.76 & 0.86 & 0.89 & 0.376 & -0.93 & 2.45 \\
\hline \multirow{3}{*}{ Level 4 } & Age & -0.25 & 0.09 & -2.78 & 0.005 & -0.42 & -0.07 \\
\cline { 2 - 9 } & Driving hours & 0.17 & 0.14 & 1.22 & 0.223 & -0.10 & 0.44 \\
\cline { 2 - 9 } & Constant & 0.21 & 0.56 & 0.38 & 0.707 & -0.89 & 1.31 \\
\hline
\end{tabular}

${ }^{1} \log$ likelihood $=-432.63$, Prob $>$ Chi2 $=0.0011$ 


\section{Discussion and Conclusion}

This study aimed to analyze driver preferences and WTP for CV features. Such knowledge is necessary for economists, policy makers, and decision makers in the automotive market to establish preferences of costumers, recognize the market's needs, and merit investment. Researchers crafted the conjoint survey - based on the literature review and successful incentives for advanced vehicles in the U.S. and offered insights into the key willingness-to-pay, adoption and attributes of CVs. As discussed in the literature review, CVs provide higher safety levels and more driving assistance abilities; therefore, the questions in the conducted conjoint survey were based on these two privileges. The results indicate that driving hours plays a privileged role among sociodemographic characteristics and driving behavior attributes of respondents. People who drive longer hours are more likely to purchase CV features. This pattern is similar to the one observed by Daziano et al. (2017) in which they found that people with longer driving ranges have a greater tendency toward using automated and emerging features in the vehicle.

The factor of age was shown to be a noticeable effect as the results showed that older people are more likely to purchase $\mathrm{CV}$ features. This result is in line with a previous study Fernandes et al. (2017) that mentioned older people may find automated and advanced features of a vehicle helpful in comfortably maintaining mobility, especially for older drivers with physical disabilities. An unexpected finding of this study was that there was no significant sign to prove people with higher income levels are more likely to purchase CVs' features. One explanation for this result can be low levels of knowledge and awareness about the effectiveness and performance of these emerging features (Zhang et al., 2019). However, the results of this study proved those who are willing to pay more money for their next vehicle are more likely to use roadway information features.

Education was the other characteristic attribute that showed a significant relationship in which people with higher education levels were more likely to purchase enhanced safety features in their next vehicle. Similarly, some studies revealed that people with higher levels of education are more eager to use automated and advanced feature in the vehicle (Daziano et al., 2017; Haboucha et al., 2017). Finally, the factor of gender was not significant in purchasing and using CVs' features. Although it was expected that men might enjoy using CVs' features more, WTP and WTU for these features did not differ by gender. This finding dovetails with the results of some previous studies (Shin et al., 2016; Zhang et al., 2019). Future studies should consider more sociodemographic characteristics and driving behavior attributes as well as more $\mathrm{CV}$ features.

\section{Acknowledgments}

The authors thank the National Transportation Center at the Morgan State University for its support. This research was supported by the Connected VehicleInfrastructure University Transportation Center at Virginia Polytechnic and State University and the University Transportation Centers Program of the U.S. Department of Transportation. The authors declare no conflict of interest. 


\section{References}

Abraham, H.; Lee, C.; Brady, S.; Fitzgerald, C.; Mehler, B.; Reimer, B.; Coughlin, J. F. 2017. Autonomous vehicles and alternatives to driving: trust, preferences, and effects of age. In Proceedings of the Transportation Research Board 96th Annual Meeting (TRB'17).

Andorka, S.; Rambow-Hoeschele, K. 2020. Urban Landscape Revolution: The Potential of Connected Vehicles and Their Impact on the Mobility Ecosystem. Springer International Publishing, 157-166.

Arvin, R.; Kamrani, M.; Khattak, A. J. 2019. How instantaneous driving behavior contributes to crashes at intersections: Extracting useful information from connected vehicle message data, Accident Analysis \& Prevention 127: 118-133.

Bansal, P.; Kockelman, K. M. 2017. Forecasting Americans' long-term adoption of connected and autonomous vehicle technologies, Transportation Research Part A: Policy and Practice 95: 49-63.

Bansal, P.; Kockelman, K. M.; Singh, A. 2016. Assessing public opinions of and interest in new vehicle technologies: An Austin perspective, Transportation Research Part C: Emerging Technologies 67: 1-14.

Bertini, R.; Wang, H.; Knudson, T.; Carstens, K. 2016. Preparing a Roadmap for Connected Vehicle/ Cooperative Systems Deployment Scenarios: Case Study of the State of Oregon, USA, Transportation Research Procedia 15: 447-458.

Bock, D. L.; Kettles, D.; Harrison, J. 2016. Automated, autonomous and connected vehicle technology assessment. Florida Solar Energy Center, FSEC Report Number: FSEC-CR-2020-16.

Choi, H.; Koo, Y. 2019. Do I have to buy it now? A vehicle replacement model considering strategic consumer behavior, Transportation Research Part D: Transport and Environment 73: 318-337.
Daziano, R. A.; Sarrias, M.; Leard, B. 2017. Are consumers willing to pay to let cars drive for them? Analyzing response to autonomous vehicles, Transportation Research Part C: Emerging Technologies 78: 150-164.

Dowling, R.; Nevers, B.; Jia, A.; Skabardonis, A.; Krause, C.; Vasudevan, M. 2016. Performance Benefits of Connected Vehicles for Implementing Speed Harmonization, Transportation Research Procedia 15: 459-470.

Ebnali, M.; Hulme, K.; Ebnali-Heidari, A.; Mazloumi, A. 2019. How does training effect users' attitudes and skills needed for highly automated driving?, Transportation Research Part F: Traffic Psychology and Behaviour 66: 184195.

Fernandes, S. C. F.; Esteves, J. L.; Simoes, R. 2017. Characteristics and human factors of older drivers: improvement opportunities in automotive interior design, International Journal of Vehicle Design 74: 167203.

Ghiasi, A.; Li, X.; Ma, J. 2019. A mixed traffic speed harmonization model with connected autonomous vehicles, Transportation Research Part C: Emerging Technologies 104: 210-233.

Gkartzonikas, C.; Gkritza, K. 2019. What have we learned? A review of stated preference and choice studies on autonomous vehicles, Transportation Research Part C: Emerging Technologies 98: 323-337.

Guerrero-Ibanez, J. A.; Zeadally, S.; ContrerasCastillo, J. 2015. Integration challenges of intelligent transportation systems with connected vehicle, cloud computing, and internet of things technologies, IEEE Wireless Communications 22: 122-128.

Haboucha, C. J.; Ishaq, R.; Shiftan, Y. 2017. User preferences regarding autonomous vehicles, Transportation Research Part C: Emerging Technologies 78: 37-49. 
Jaffry, S.; Apostolakis, A. 2011. Evaluating individual preferences for the British Museum, Journal of Cultural Economics 35(1): 49-75.

Jiang, Y.; Zhang, J.; Wang, Y.; Wang, W. 2018. Capturing ownership behavior of autonomous vehicles in Japan based on a stated preference survey and a mixed Logit model with repeated choices, International Journal of Sustainable Transportation 13(10): 788-801.

Kidando, E.; Moses, R.; Ghorbanzadeh, M.; Ozguven, E. E. 2018. Traffic Operation and Safety Analysis on an Arterial Highway: Implications for Connected Vehicle Applications. In Proceedings of the $21^{\text {st }}$ International Conference on Intelligent Transportation Systems (ITSC), 2753-2758.

Kopelias, P.; Elissavet, D.; Vogiatzis, K.; Skabardonis, A.; Zafiropoulou, V. 2020. Connected \& Autonomous Vehicles - Environmental Impacts - A review, Science of The Total Environment 712: 135237.

Kyriakidis, M.; Happee, R.; De Winter, J. C. F. 2015. Public opinion on automated driving: Results of an international questionnaire among 5000 respondents, Transportation Research Part F: Traffic Psychology and Behaviour 32: 127-140.

Li, L.; Liu, Y.; Wang, J.; Deng, W.; Oh, H. 2016. Vehicle mobility driven by traditional drivers versus connected drivers, Wireless Networks 22: 1891-1900.

Liu, P.; Guo, Q.; Ren, F.; Wang, L.; Xu, Z. 2019a. Willingness to pay for self-driving vehicles: Influences of demographic and psychological factors, Transportation Research Part C: Emerging Technologies 100: 306-317.

Liu, P.; Yang, R.; Xu, Z. 2019b. Public Acceptance of Fully Automated Driving: Effects of Social Trust and Risk/Benefit Perceptions, Risk Analysis 39: 326-341.

Medenica, Z. 2019. Human Machine Interaction. In: MIUCIC, R. (ed.) Connected Vehicles: Intelligent Transportation Systems. Cham: Springer International Publishing.
Motamedi, S.; Wang, P.; Chan, C. Y. 2018. User Acceptance and Public Policy Implications for Deployment of Automated Driving Systems. Institute of Transportation Studies, Berkeley.

Narla, S. R. 2013. The evolution of connected vehicle technology: From smart drivers to smart cars to selfdriving cars, Ite Journal 83: 22-26.

National Highway Traffic Safety Administration. 2018. Automated Vehicles for Safety. United States Department of Transportation.

Outay, F.; Kammoun, F.; Kaisser, F.; Atiquzzaman, M. 2017. Towards Safer Roads through Cooperative Hazard Awareness and Avoidance in Connected Vehicles. In Proceedings of the $31^{\text {st }}$ International Conference on Advanced Information Networking and Applications Workshops (WAINA), 208-215.

Payre, W.; Cestac, J.; Delhomme, P. 2014. Intention to use a fully automated car: Attitudes and a priori acceptability, Transportation Research Part F: Traffic Psychology and Behaviour 27: 252-263.

Razaob, A.; Mansor, M.; Khamis, N.; Kassim, K. 2019. Willing of public to purchase and understanding of pedestrian AEB system in Malaysia. IOP Conference Series: Materials Science and Engineering, IOP Publishing, 012062.

Sahebi, S.; Nassiri, H. 2017. Assessing Public Acceptance of Connected Vehicle Systems in a New Scheme of Usage-Based Insurance, Transportation Research Record 2625: 62-69.

Sawtooth Software. 2009. ACBC Technical Paper. Sawtooth Software Technical Paper Series, 1-21.

Sayer, J. R.; Leblanc, D. J.; Mefford, M. L.; Devonshire, J. 2007. Field test results of a road departure crash warning system: driver acceptance, perceived utility and willingness to purchase. In Proceedings of the Fourth International Driving Symposium on Human Factors in Driver Assessment, Training and Vehicle Design, 77-83.

\section{jitte 227}


Schoettle, B.; Sivak, M. 2014.A survey of public opinion about connected vehicles in the U.S., the U.K. and Australia. In Proceedings of the International Conference on Connected Vehicles and Expo (ICCVE), 687-692.

Sener, I. N.; Eluru, N.; Bhat, C. R. 2009. An analysis of bicycle route choice preferences in Texas, US. Transportation 36: 511-539.

Shabanpour, R.; Golshani, N.; Shamshiripour, A.; Mohammadian, A. 2018. Eliciting preferences for adoption of fully automated vehicles using bestworst analysis, Transportation Research Part C: Emerging Technologies 93: 463-478.

Shabanpour, R.; Mousavi, S. N. D.; Golshani, N.; Auld, J.; Mohammadian, A. 2017. Consumer preferences of electric and automated vehicles. In Proceedings of the $5^{\text {th }}$ IEEE International Conference on Models and Technologies for Intelligent Transportation Systems (MT-ITS), 716-720.

Shin, H. S.; Callow, M.; Dadvar, S.; Lee, Y. J.; Farkas, Z.A. 2015. User Acceptance and Willingness to Pay for Connected Vehicle Technologies: Adaptive ChoiceBased Conjoint Analysis, Transportation Research Record 2531: 54-62.

Shin, H. S.; Callow, M.; Farkas, Z. A.; Lee, Y.J.; Dadvar, S. 2016. Measuring User Acceptance of and Willingness-to-Pay for CVI Technology. Connected Vehicle/Infrastructure University Transportation Center (CVI UTC).
Shin, H. S.; Farkas, Z. A.; Nickkar, A. 2019. An Analysis of Attributes of Electric Vehicle Owners' Travel and Purchasing Behavior: The Case of Maryland, International Conference on Transportation and Development, June 9-12, 2019, Alexandria, Virginia.

United States Census Bureau. 2017. U.S. Census Data and Statistics. Available from internet: $<$ https://data. census.gov/cedsci/>.

Van Themsche, S. 2016. E-Mobility likely winners and losers. The Advent of Unmanned Electric Vehicles. Springer.

Wang, H.; You, F.; Chu, X.; Li, X.; Sun, X. 2019. Research on Customer Marketing Acceptance for Future Automatic Driving - A Case Study in China City, IEEE Access 7: 20938-20949.

Zhang, Y.; Wu, C.; Qiao, C.; Hou, Y. 2019. The effects of warning characteristics on driver behavior in connected vehicles systems with missed warnings, Accident Analysis \& Prevention 124: 138-145.

Zmud, J.; Sener, I. N.; Wagner, J. 2016a. Consumer acceptance and travel behavior: impacts of automated vehicles. Texas A\&M Transportation Institute.

Zmud, J.; Sener, I. N.; Wagner, J. 2016b. Self-Driving Vehicles: Determinants of Adoption and Conditions of Usage, Transportation Research Record 2565: 57-64. 